\title{
Sexual and reproductive health of South Asians in the UK: an overview
}

\author{
Catherine Griffiths, Audrey Prost, Graham Hart
}

\section{Introduction}

People from minority ethnic groups, forming approximately $8 \%$ (4.6 million people) of the population in the UK, are at a disproportionate risk of social exclusion and health inequality. ${ }^{1}$ The reproductive and sexual health of some of these groups has been identified as a particular area of concern because many are at a higher risk of teenage pregnancy and sexually transmitted infections (STIs) than the general population. ${ }^{2,3}$ Service access has also been found to be poor among many. ${ }^{4}$ In order to inform and develop appropriate sexual health interventions and improve service access there is a need to improve our understanding of the sexual behaviours and attitudes of different minority ethnic groups and the reasons for poor health outcomes.

Ethnic categorisation and the use of ethnicity as a variable and within health research is an ongoing area of debate. People are often allocated to racial or ethnic groups arbitrarily. Categories of ethnic group are sometimes undefined and the use of terms inconsistent. ${ }^{5}$ Broad ethnic/racial classification such as Asian, South Asian, black African, and so on mask the rich diversity of religions, cultures and languages that exist between and within particular ethnic groups. They also mask important socioeconomic differences that may underpin health inequality. Ethnic groups are generally characterised by a sense of belonging or group identity, sometimes derived from a sharing of place of birth of oneself or one's parents. They are often perceived as sharing a common culture, a set of behaviours and beliefs, determined by upbringing and choice. ${ }^{5}$ However, ethnic groups are also determined by social pressures and psychological needs and are therefore fluid and dynamic.5 Given this, ethnicity or ethnic identity may indeed change over time for individuals and mean different things to different people. It is argued that this makes a universal taxonomy impossible. 6 However, if researchers want to be able to compare research findings there is a need for some level of consistent classification. ${ }^{6}$ To achieve this, Bhopal and Senior emphasise the need for researchers to explicitly state how their ethnic classifications were made in order to facilitate comparison. They also suggest exercising caution in generalising results across and within ethnic groups, and to consider the relative importance of other socioeconomic, cultural and lifestyle variables in explaining variations in health. ${ }^{7}$

J Fam Plann Reprod Health Care 2008; 34(4): 251-260

(Accepted 6 December 2007)

Centre for Sexual Health and HIV Research, University

College London, London, UK

Catherine Griffiths, MSc, BSc, Research Fellow

Graham Hart, BA, PhD, Centre Director

MRC Social and Public Health Sciences Unit, Glasgow, UK Audrey Prost, BA, PhD, Research Fellow

Correspondence to: Ms Catherine Griffiths, Centre for Sexual Health \& HIV Research, Research Department of Infection \& Population Health, University College London, Mortimer Market Centre, Off Capper Street, London WC1E 6JB, UK.

E-mail: cgriffiths@gum.ucl.ac.uk
In the UK, particularly in the recording of demographic data and more often than not in research, 'Asian' is the most widely accepted current term used to refer to people of South Asian background, namely people of Indian, Pakistani and Bangladeshi descent/ethnicity. ${ }^{8}$ However, this is misleading as Asian can refer to people not only from the Indian subcontinent but also the Far East and Southeast Asia. This paper focuses on Indians, Pakistanis and Bangladeshis and therefore uses the term 'South Asian' when collectively referring to these ethnic groups. However, even this label fails to capture all self-defined ethnic identity. ${ }^{9}$ When referring to research findings, this review uses the terms as used and described by the authors and attempts where possible to clarify any broad categorisation and to present results by Bangladeshi, Indian or Pakistani origin.

Until recently there has been a dearth of research into the sexual and reproductive health of South Asians in the UK. This is perhaps surprising given that they constitute the largest ethnic group in the UK, ${ }^{8}$ but may in part be due to the reluctance of researchers to explore an issue considered by many South Asian communities to be extremely sensitive and which carries with it a number of cultural and faith taboos. It may also be because there is limited epidemiological data showing sexual ill health among South Asians and they are therefore considered low risk relative to other population groups. The aim of this review is to illuminate and collate our understanding to date of the sexual and reproductive health needs of Bangladeshis, Indians and Pakistanis in the UK.

\section{Methods}

A literature search was carried out in MEDLINE and the Web of Science using science citation index, social sciences citation index and arts and humanities citation index. Combinations of the following words were searched for: 'Asian', 'South Asian', 'sexual health', 'contracept*', sexually transmitted infection*, HIV, Indian*, Pakistani* and Bangladeshi*. Google Scholar ${ }^{\mathrm{TM}}$ and the Department of Health were searched for relevant reports using the same terms. The bibliographies of retrieved articles/reports were then searched for further references. Literature was limited to published research conducted in the UK between January 1990 and June 2007. All types of studies were considered for inclusion with no limit on study design. Twenty-four research studies were found which focused on South Asians generally or on one of the specific South Asian ethnic groups (Bangladeshi, Pakistani, Indian). Some of the studies reviewed had South Asians as part of their overall study sample, which included other ethnic groups. Studies were divided into four main areas: sexual knowledge and behaviour, STIs and HIV, contraceptive behaviour, and service access.

\section{Findings and discussion}

All included studies were descriptive or observational and included both quantitative and qualitative research. A summary of the studies reviewed can be found in Tables 1 and 2 .

Sociodemographic overview

Based on 2001 census data, Indians followed by Pakistanis 







\begin{tabular}{|c|c|c|c|c|c|c|c|}
\hline & 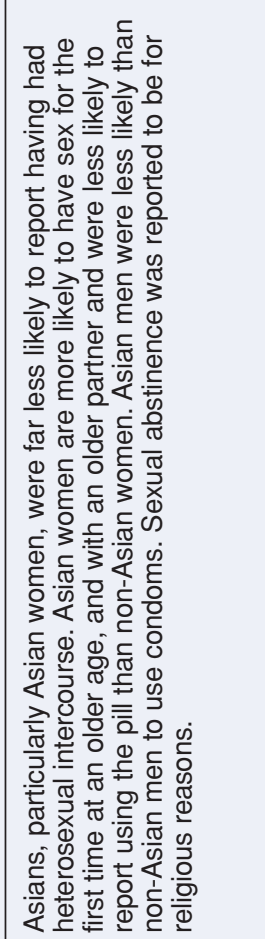 & 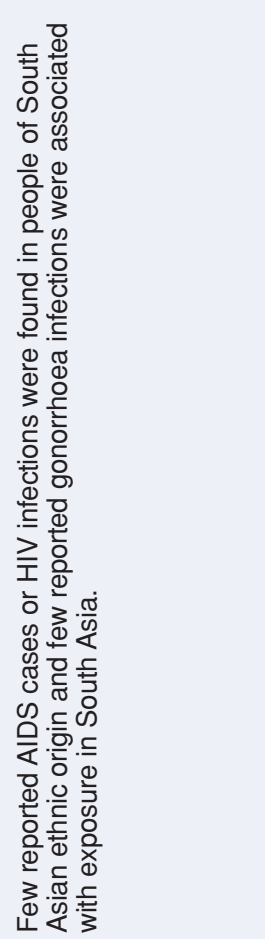 & 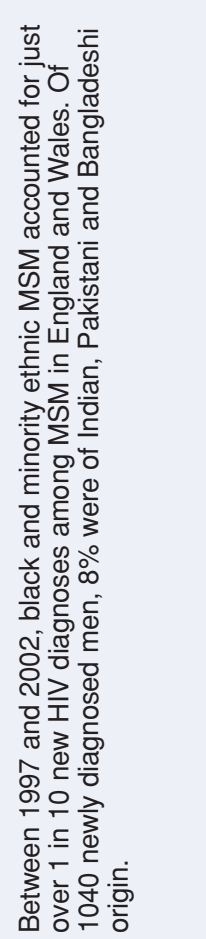 & 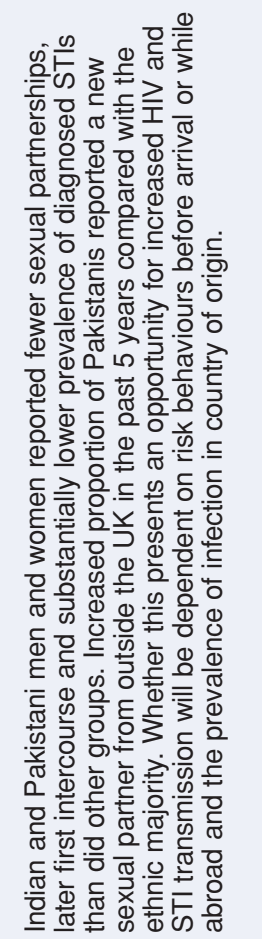 & 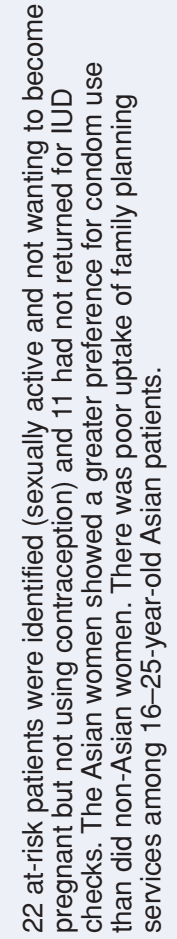 & 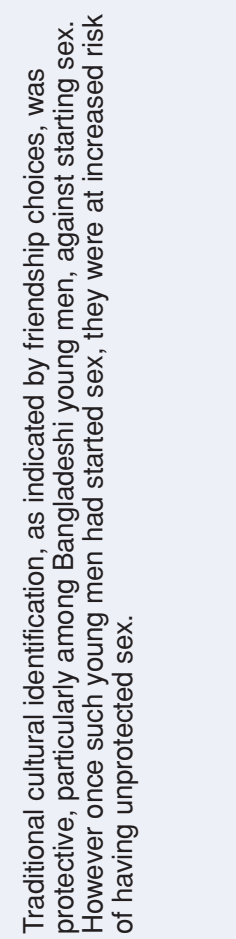 & 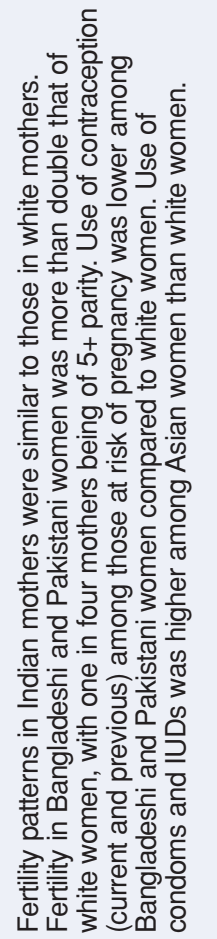 \\
\hline & 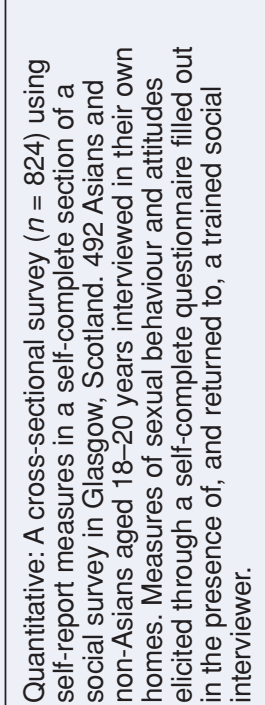 & 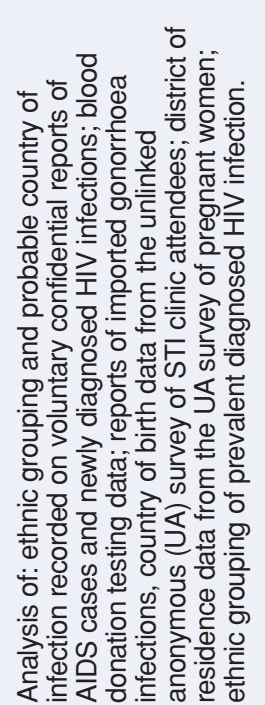 & 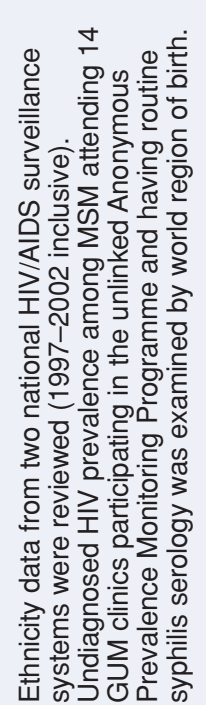 & 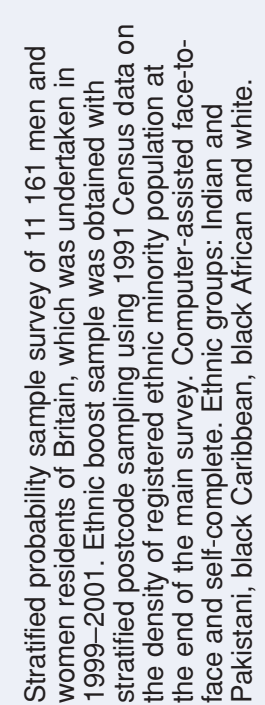 & 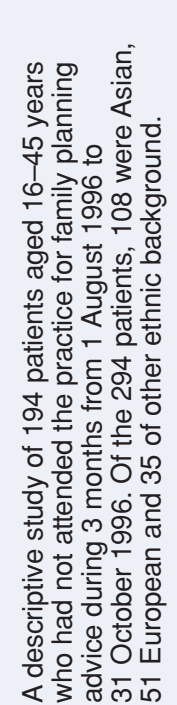 & 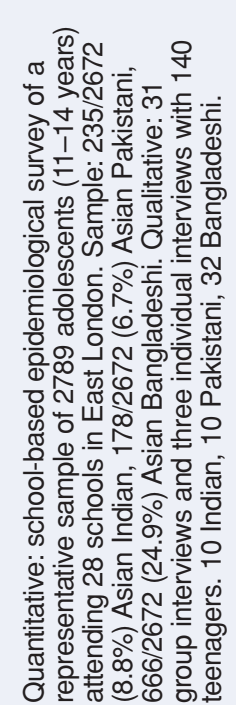 & 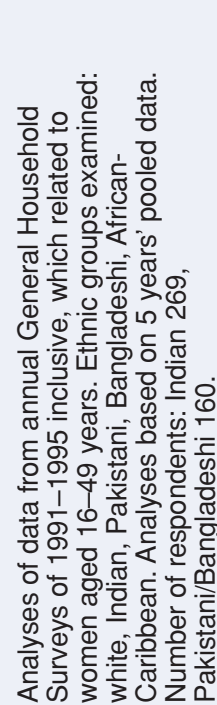 \\
\hline$\stackrel{n}{\frac{E}{\alpha}}$ & 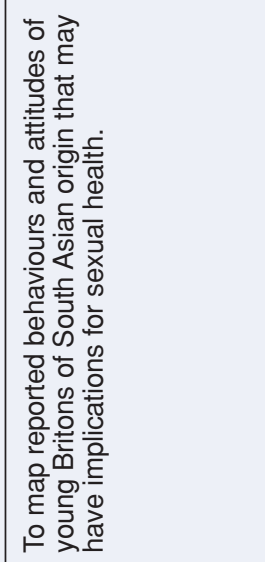 & 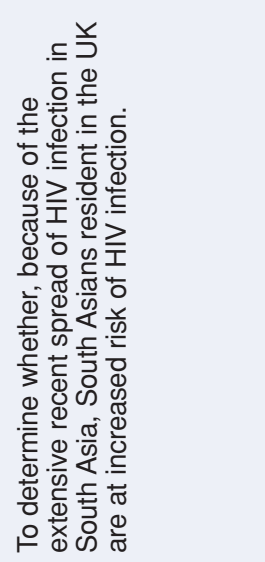 & 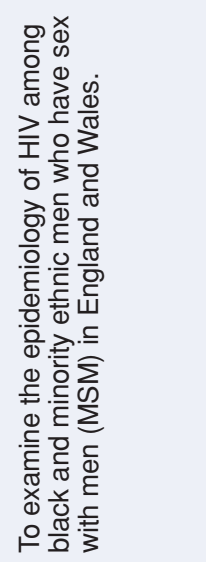 & 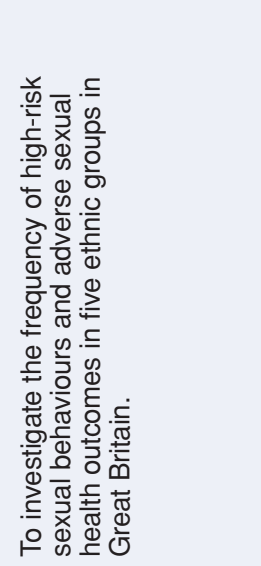 & 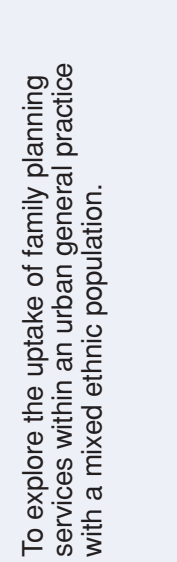 & 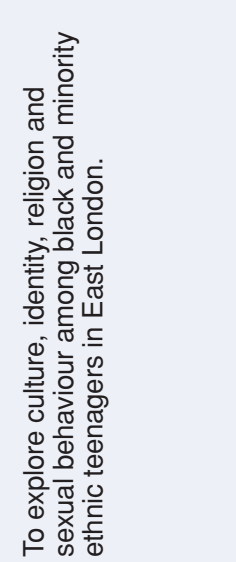 & 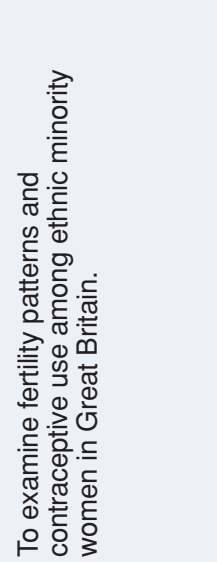 \\
\hline & : & ه্. & 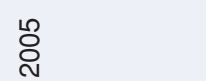 & ֻั & $\begin{array}{l}\text { o } \\
\stackrel{\circ}{\sigma}\end{array}$ & ஜి & 용 \\
\hline & 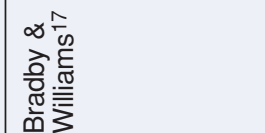 & 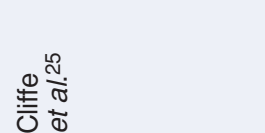 & 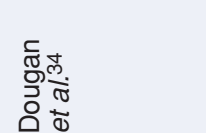 & 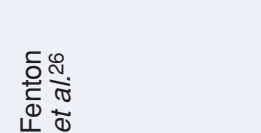 & & 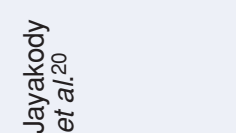 & \\
\hline
\end{tabular}




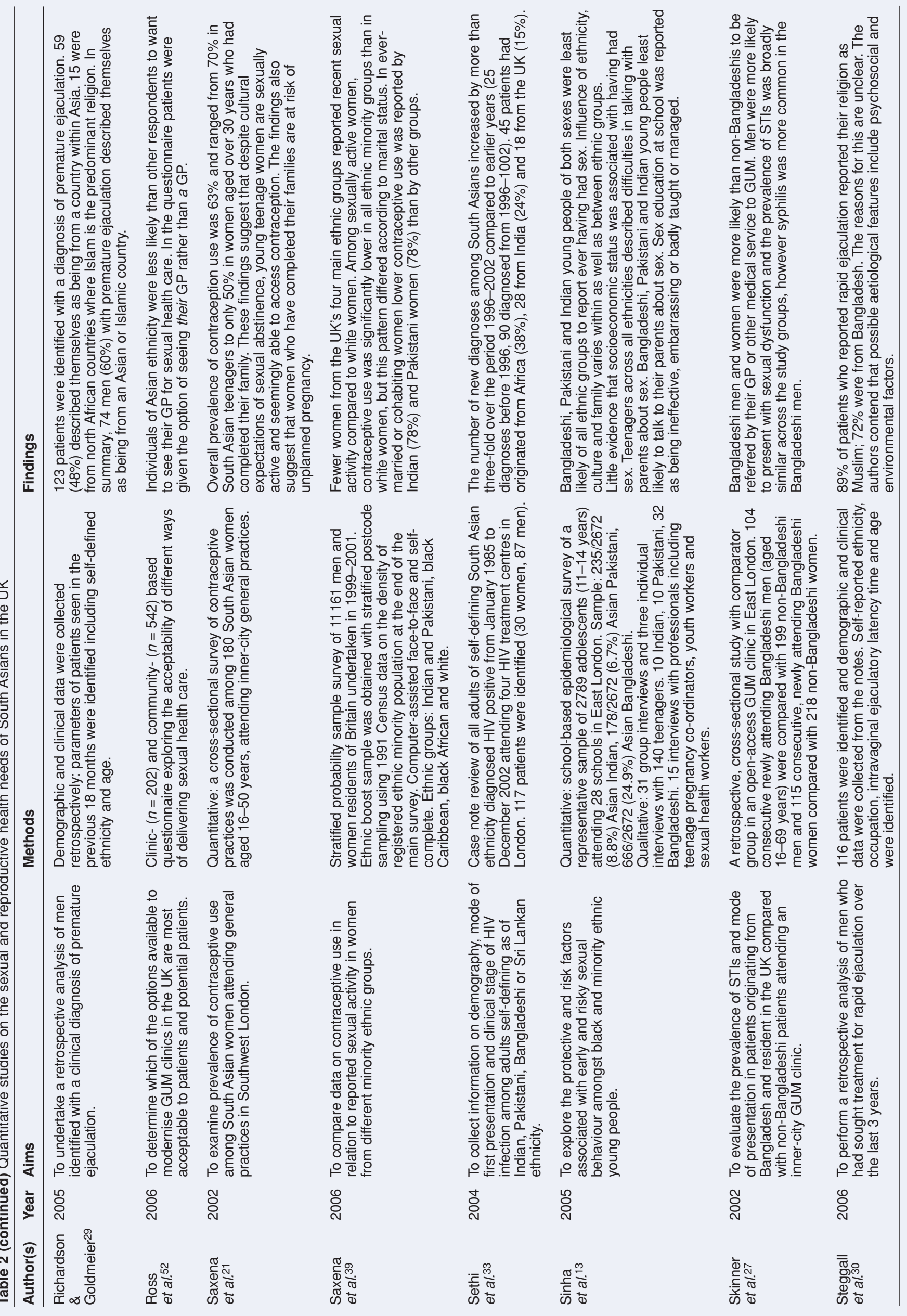




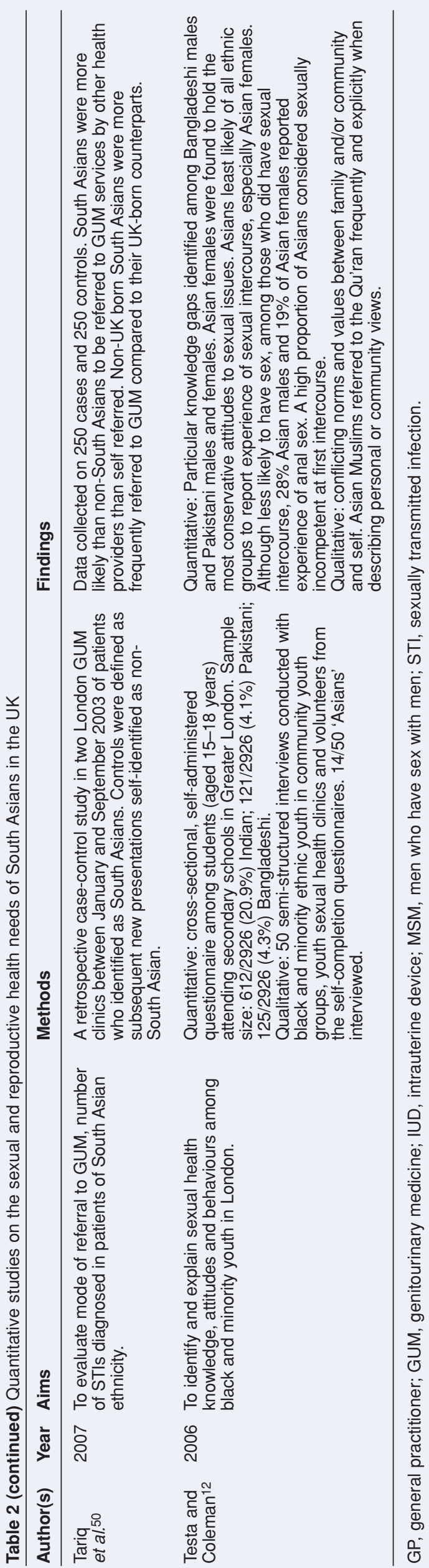


are the largest ethnic groups in the UK, representing $22.7 \%$ and $16.1 \%$, respectively, of the British non-white population. ${ }^{8}$ Bangladeshis are the smallest of the three main South Asian communities and make up just over 6\% of all ethnic minorities. South Asians thus account for almost half of Britain's ethnic minorities and over $96 \%$ live in England itself. Many are at a disproportionate risk of social exclusion. Pakistanis and Bangladeshis are more likely than other ethnic groups to be living on low incomes and have high rates of unemployment. Over $40 \%$ of Bangladeshi men aged under 25 years are unemployed. They are also the most likely to have no qualifications. ${ }^{10}$ Conversely, Indians have higher educational achievement and lower levels of unemployment. South Asian households are larger than households of any other ethnic group and Bangladeshis (38\%) and Pakistanis (35\%) have high proportions of young people aged under 16 years, which is almost double the proportion of white British people (19\%). Pakistani and Bangladeshi men and women in England and Wales have also reported the highest rates overall of being in 'not good' health. ${ }^{11}$

\section{Sexual knowledge and behaviour}

Research exploring the sexual health knowledge and behaviour of South Asians in the UK is mostly focused on unmarried, young people (aged 13-25 years) in defined geographical areas. These studies, including both survey and/or qualitative data, have consistently demonstrated relatively poor sexual health knowledge among young South Asians. ${ }^{12-14}$ Testa and Coleman found poor knowledge in terms of STI name, symptom recognition and means of prevention and identified particular gaps among Bangladeshi males and Pakistani males and females..$^{12}$ French et al. also identified particular knowledge gaps among Bangladeshi and Indian young people and found that school was often the only source of sex and relationships education available as sex was almost never discussed at home. ${ }^{14}$ However, sex and relationships information provided in schools has been found to take little account of faith or cultural issues relevant to these young people $\mathrm{e}^{15}$ and is seldom considered to be a useful source of information. ${ }^{13-16}$

South Asian young men and women are less likely than their peers to report experience of sex. ${ }^{12,13}$ However, there appear to be gender differences, with young men reporting more sexual activity than their female counterparts. ${ }^{14,17}$ Testa and Coleman also found that among those who had had sex, $28 \%$ of males and 19\% of females reported experience of anal sex. The reasons for this are unclear and were not presented in the qualitative data. ${ }^{12}$ Elsewhere, practitioners have alluded to this, suggesting that though these young South Asians may not be engaging in sexual activity conventionally defined as sex, there may be other behaviours occurring that could compromise their sexual health (e.g. anal sex, heavy petting, mutual masturbation and oral sex). ${ }^{13}$ Approximately $25 \%$ of sexually active Asian males in Testa and Coleman's survey sample reported two or more partners in the last 6 months ${ }^{17}$ and contraceptive and condom use has been found to be poor. ${ }^{12,14}$

In 2001, a measure of sexual competence for first intercourse was constructed based on four variables relating to circumstance: regret, willingness, autonomy and contraception. ${ }^{18}$ A similar measure was derived in Testa and Coleman's study based on a number of events in relation to first ever sexual intercourse (reasons such as love, drunk, etc., relationship status, timing, willingness, contraception). Based on this a very high proportion of Asian males (83.2\%) and females $(80.9 \%)$ were considered sexually incompetent. ${ }^{12}$ These findings indicate that though low in numbers, the South Asians who are having sex have distinct sexual health needs.

Many data are collected from young people of school age who are likely to be resident at home. Many Asian young people's sexual behaviour, particularly that of females, is influenced by home contexts where cultural traditions, religious obligations, family loyalties and community expectations prevent mixing with the opposite sex and promote abstinence norms ${ }^{19,20}$ However, Hennink found that once young women (18-30 years) leave home they experience an independence often involving relationships and sexual activity. ${ }^{19}$ She suggests that these young women become influenced by new peer groups from a range of non-Asian backgrounds and that their first sexual experience is often with non-Asian partners. Another study has also found some unmarried South Asian women to be sexually active. ${ }^{21}$ This suggests the beginning of behaviour changes, with some South Asian young people now having attitudes and behaviours that differ from their parents. ${ }^{19}$ This transition from the behavioural norms of their parents' generation has been found elsewhere. ${ }^{14,22}$

The strength of research to date is in the triangulation of methods used to capture sexual health data including social surveys with sexual health components, ${ }^{17}$ school-based surveys coupled with community-based qualitative exploration, ${ }^{12,23}$ and exploratory, descriptive communitybased qualitative studies. 14,19,22 However, there are several limitations that need consideration. With collection of sensitive data there are issues of reporting bias and social desirability. This may be prominent in South Asian people's responses where behaviour considered culturally unacceptable may be underreported for fear of adverse judgement. ${ }^{24}$ That two of the large surveys were conducted in London only also needs consideration. The consistency of some findings across geographical areas (e.g. Glasgow, Greater London, Birmingham, Manchester) is somewhat reassuring. However, we need to consider possible differences between young people of the same ethnic/cultural background who live in different geographical regions in the UK. Communities often evolve differently in different places and where local ethnic population profiles/compositions vary, friendship group formation and sexual mixing may vary with implications for sexual behavioural risk. A final problem is the collation of ethnic groups into broad categories such as South Asian. This was unavoidable in some studies as low overall numbers of sexually active South Asians meant that analysis of sexual behaviour findings by Indian, Bangladeshi and Pakistani ethnicity were not possible. However, categorisation masks the heterogeneity of behaviours, cultures, religions, languages and beliefs that may exist between and within ethnic groups. We need to be cautious in applying the behaviours and patterns reported above to all South Asians or to all individuals within ethnic subgroups and be aware that behaviours may well be underreported.

Some authors have found that young people's experiences can be better understood by religious, cultural and friendship group contexts rather than by ethnicity. For example, Testa and Coleman ${ }^{12}$ found that Muslims from a diverse range of ethnicities shared similar sexual attitudes, and Muslim Asians referred to the Qu'ran frequently and explicitly when describing their personal or community views. Another study found that having traditional friendship choices (friends from the same cultural background) was protective of starting sex in young men, particularly among Bangladeshis. However, it was also 
associated with increased risk of not using protection when sexually active. ${ }^{20}$ These findings need further exploration in order to understand the association. In using markers of cultural/ethnic identity to explain sexual behaviour we must also be cautious of problematising culture and of ignoring the socioeconomic root causes of health disadvantage. 20

\section{STIs and HIV}

Though increasing data are available on the sexual behaviour of South Asians in the UK, there are limited data reporting sexual health outcomes such as STIs. Ethnic origin is not routinely collected in genitourinary medicine (GUM) clinics and there are the aforementioned limitations of definitions and categorisations of ethnicity. Existing data have generally shown lower rates (actual and reported) of STIs in South Asian groups compared to white groups. ${ }^{25,26}$ One GUM clinic study found similar rates of STIs in Bangladeshi attendees as non-Bangladeshi attendees, though this was mainly among individuals born in Bangladesh. ${ }^{27}$ Bangladeshi men were also more likely than non-Bangladeshis to present with sexual dysfunction, and both men and women were more likely to have been referred to GUM from general practice and family planning services. Sexual dysfunction and premature ejaculation among some South Asian and Muslim men has been found elsewhere. ${ }^{28-30}$ This does not indicate that South Asian or Muslim men have higher rates of sexual dysfunction, rather they may be more concerned about it and seek treatment as compared to other groups..$^{30}$ The reasons behind the sexual dysfunction remain unclear, though possible influences include psychosocial, familial and genetic factors. 'Dhat syndrome' or semen loss has also been widely reported as a sexual concern among Indian men. ${ }^{31}$

In 2005 in England, Wales and Northern Ireland, 1.4\% (321/22 324) of heterosexuals living with diagnosed HIV were of Indian, Pakistani or Bangladeshi ethnicity. ${ }^{2}$ This rate is low relative to some other population groups, however some suggest that increasing HIV incidence in South Asia combined with migration due to strong familial, social and business links between South Asians in the UK and those of the South Asian region may be cause for concern. ${ }^{26,32}$ There are, however, no data to verify the direct impact of migration on HIV rates among South Asians to date, although national surveillance suggests increasing infection, and a retrospective case note review across four treatment centres in London found a three-fold increase in new diagnoses between 1996 and 2002.33 Recent years have seen a substantial rise in the number of new HIV diagnoses in men who have sex with men (MSM). While the ethnicity of heterosexually acquired HIV has been examined in detail, there has been little consideration of HIV among black and minority ethnic MSM. A recent study found that 12\% (1040/8861) of all newly diagnosed HIV among MSM was in black and minority ethnic individuals. Of the $1040,8 \%$ were Indian/Pakistani/Bangladeshi. ${ }^{34}$

\section{Contraceptive behaviour}

Improving contraceptive uptake and access to services and information among many black and minority ethnic women is part of a national initiative to reduce unintended pregnancy. 35 There is also a need to ensure individuals have equitable access to a full range of contraceptive methods. ${ }^{36}$ Contraceptive surveys conducted in the 1970 s and 1980s did not provide data on ethnic background and, in the few studies where ethnicity was referred to, South Asians constituted less than $5 \%$ of the study population. 37 Other studies conducted in the 1980s have involved small, select populations from family planning clinics or ante/postnatal departments and found varying degrees of contraceptive use and variation in the type used. ${ }^{37}$ More recent studies have also shown varying levels of contraceptive use among South Asian women but usage has consistently been shown to be lower than for their white counterparts. ${ }^{38,39}$ Some of this survey data does, however, rely on pooled data over several years because of small sample sizes and may therefore have underestimated contraceptive use due to a lack of information about sexual activity. ${ }^{39}$

South Asian women, particularly Bangladeshi and Pakistani women, have higher parity than white women and often continue childbearing to older ages. ${ }^{38,40}$ One study found that about a third of married women and half of women over 30 years of age who said that they had completed their families were not using contraception and were therefore at risk of unplanned pregnancy. ${ }^{21}$ In contrast, in the same study, unmarried women (mainly teenagers) were more likely to be using contraception than married women, which indicates that despite cultural expectations of no premarital sex, some young women are sexually active and able to access contraception. However, the sample in this study is small and taken from a general practice and therefore excludes women unable to access health services altogether, including contraception. These findings also contrast with other studies of young sexually active Asians, which have found relatively poor contraceptive use. ${ }^{14,17}$ In terms of type of contraception, data from the National Survey of Sexual Attitudes and Lifestyles II (Natsal 2000) (39 $^{3}$ found that South Asian women were less likely than white women to report using hormonal contraception and permanent methods and were more likely to use barrier methods, which is consistent with some earlier studies. ${ }^{41-44}$ Similarly, Raleigh et al. ${ }^{38}$ found that the use of condoms and intrauterine devices was higher among Bangladeshi and Pakistani women than other groups. What appears to be consistent is that many Asian women are using less reliable methods of contraception.

Hennink and colleagues 45,46 found it more meaningful to group women into educational/professional categories within which they displayed relatively homogenous patterns of family planning behaviour rather than by cultural or ethnic group. They found it difficult to identify the family planning needs and service delivery implications for such a diverse group of women as is covered by the term 'South Asian'. Most non-professional women were found to have little knowledge of contraception until after marriage or their first birth. Condom use was then the most reported contraception among these women. Most of these women's decisions were influenced by (extended) family (particularly husbands and mother-in-laws) and religious/cultural expectations of fertility. These women were also found to have difficulties in communicating with health professionals due to poor English, literacy and a lack of confidence. Conversely, professional women were more likely to have knowledge of contraception and to have used some method throughout their childbearing years (typically the pill). They also cited personal, practical or economic considerations in their fertility decisions. Women's opinions about the influence of religion on contraceptive use were placed on a continuum whereby contraceptive use was perceived as forbidden, discouraged or acceptable. For non-professional Muslims contraception was considered 'forbidden' and these women were not likely to use contraception through their childbearing years, however for professional women it was perceived as 'discouraged' and did not affect use.

There is a paucity of research reporting on abortion 
among South Asians in the UK. Studies have in the main looked at attitudes to abortion rather than rates among particular population groups. ${ }^{14,47}$ Other work from outside the UK suggests that induced abortion does occur among some South Asian populations and that the reasons for it can be diverse. Examples include reasons such as desire for educational attainment, ${ }^{48}$ birth spacing and lack of contraceptive knowledge 48 and intimate partner violence. 48

\section{Access to services}

Although there is Department of Health guidance for developing contraception and sexual health advice services for black and minority ethnic young people, ${ }^{35}$ there is little research exploring sexual health service use specifically among South Asians in the UK. National routine data collected in family planning and GUM clinics does not include ethnic origin of attendees. The work that has been conducted is qualitative and in the main explores awareness, preference and barriers to service access. Poor awareness of specialist sexual health services (i.e. GUM) is a common theme across studies ${ }^{4,14}$ and many South Asians have expressed the need for more accessible information about services. ${ }^{4}$ A case-control study in two London GUM clinics found that South Asians were significantly more likely than controls to have been referred by other medical services rather than self-referred, perhaps reflecting the lack of awareness of services or barriers to access. ${ }^{50}$ In terms of service preference, one study found general practice to be the most preferred setting for sexual health care among Indians, Pakistanis and Bangladeshis. However, in another study Asians were less likely than other ethnic groups to prefer general practice, though there is no description in this study of 'Asian'. 51 That the reason for a visit can be concealed and that GPs are local and conveniently situated are the cited advantages of general practice for sexual health. ${ }^{4}$ However, concerns about the confidentiality of GPs are prevalent, particularly among Indians. Family GPs, often of the same ethnic background, are perceived as potentially disclosing information to other family members. ${ }^{4,14}$ Having a service provider of the same cultural or religious background has also been found to be problematic, ${ }^{14,16}$ with some people feeling that this provider may judge them if their behaviour is seen as deviating from cultural or religious norms. Moreover, it is perceived that a person of the same ethnic and cultural background may have links with the local community, thus undermining feelings of confidentiality and privacy. Having a provider that understands cultural norms has been found to be important however. Matched gender is also important, particularly for females. ${ }^{4}$

Some South Asians have been found to prefer GUM services because they are specialised, ${ }^{4}$ however stigma and anxiety of being seen remain consistent themes. In general, for many South Asian communities, faith and cultural values prohibit premarital sex and therefore dialogues around sexual health are deemed unnecessary and irrelevant. ${ }^{16}$ Discussion of sex implies a lack of respect for the person you are talking to, and being seen accessing any related service would have an impact on one's standing in the community and on marriage prospects for the family. Concepts of individual and collective honour or izzat and shame are central and act as a central framework of social control, encouraging the masking of shameful behaviour and promoting public conformity. 32 This means that many individuals in need of sexual health care may fail to access appropriate services. It also means that services need to find ways to enable people from South Asian communities to better access them. This is particularly important for
South Asians who are recently immigrated and/or who do not speak English and also applies to contraceptive/family planning services. 52

\section{Summary and conclusions}

This overview highlights a range of research (1990-June 2007) and sexual health promotion needs for Bangladeshis, Indians and Pakistanis in the UK. Although there are subtle differences between each ethnic group, there is evidently a need among all for improved sexual health knowledge and awareness. Contraceptive use is poor among South Asian young people, and married women who have completed their families have been found to use no contraception or less reliable methods thus placing them at risk of unintended pregnancy. Research suggests there are few sexually active unmarried young people, however those that are have been found to have distinct sexual health needs. Young men in particular have been found to have two or more partners in the last 6 months and report poor condom use and there is some evidence of anal sex, possibly as a means of contraception, among both males and females. This requires further investigation.

A prominent gap in the research to date is an understanding of the behaviours and needs of young unmarried South Asians aged 16-30 years. Work has in the main focused on school age people or married women. However, one study suggests that once some South Asian unmarried young women leave home they begin to engage in sexual relationships before marriage. This exploratory work needs to be built upon and the needs of men aged 16-30 years explored also. The context of these young people's lives requires closer investigation if we are to better inform appropriate sexual health promotion initiatives. For example, we need to better understand who they spend time with, the subcultures they adhere to and that influence them, their choice of partnerships and sexual mixing, the role of drugs and alcohol as well as other social, cultural and economic factors that may impact on the behaviour of young South Asians in the UK today. We need to explore further the ways in which many young people balance the more 'traditional' cultural perspective of their parents and community with the perspectives and norms of the wider society and non-Asian peers and the implications this has for behavioural risk and sexual health.

School is not perceived by young people to be a useful source of information, and sex and relationships education (SRE) has been found to take little account of faith and culture. If we are to improve sexual health knowledge among South Asians in the UK there is an urgent need to address the content and delivery of SRE across the UK, as many young people are unable, due to faith and cultural constraints, to get information at home. Given the importance of faith and cultural values to many South Asian communities, it is also crucial that this sexual and reproductive health information, whether provided within or outside of school settings, takes account of these issues and attempts to involve parents and community leaders.

There appears to be a paucity of recent work (with the exception of Hennink and colleagues) qualitatively exploring contraceptive decisions and fertility issues for South Asian women and their access to appropriate care, including issues around abortion. Many women learn about contraception at first birth and it has therefore been suggested that midwives and health visitors have an important role in the dissemination of culturally appropriate information. It is also important to increase awareness among all health professionals of the context in which these women make reproductive choices and the complex range of influences (cultural, familial) on their 
family planning and sexual behaviour. Health promotion material also needs to be culturally sensitive and linguistically appropriate. The fpa (formerly the Family Planning Association) has gone some way towards addressing this and has produced specific sexual health promotion material for South Asian women.53 General practice has been shown in some cases to be the preferred service for sexual and reproductive health needs among South Asians, and GUM attendees have often been referred from general practice and family planning services. This suggests the need for non-GUM providers to consider sexual health issues and STIs in the differential diagnosis of presentations among their South Asian patients. Again there is the need to ensure professionals understand the different cultural and faith influences that shape sexual and health seeking behaviour among Bangladeshis, Indians and Pakistanis.

Very little published research has been conducted with South Asian MSM in the UK, though much culturally and linguistically appropriate support and health promotion work for these men is carried out by Naz Project London, an HIV, AIDS and sexual health agency for black and minority ethnic communities. ${ }^{54}$ Like Naz Project London, there are a growing number of organisations across the UK conducting sexual health work with black and minority ethnic communities and specifically with South Asians. However, documentation or 'grey literature' of such work can be difficult to obtain. One of the main limitations of this review is that it does not cover 'grey literature' or local reports but focuses on peer-reviewed published studies. There is, however, a real need to improve ways of sharing good practice and learning across organisations, and of collating and reviewing/evaluating work that is being carried out but that may not be published. The Teenage Pregnancy Unit in 2002 with the document Diverse Communities: Identity and Teenage Pregnancy, A Resource for Practitioners 55 is one example of such collation but this now needs to be expanded and updated. Finally, in order to facilitate comparison and ensure that research findings can be taken forward to inform practice it is important that researchers continue to explicitly and consistently state how their ethnic classifications are made and, where possible, avoid generalising results across and within ethnic groups.

\section{Statements on funding and competing interests}

Funding Catherine Griffiths is funded by a Personal Award Scheme Researcher Development Award from the National Institute for Health Research.

Competing interests None identified.

References

1 Social Exclusion Unit. Minority Ethnic Issues in Social Exclusion and Neighbourhood Renewal. London, UK: The Stationary Office, 2000

2 Health Protection Agency (HPA). Mapping the Issues - HIV and Other Sexually Transmitted Infections in the United Kingdom 2005. The UK Collaborative Group for HIV and STI Surveillance. London, UK: HPA, 2005.

3 Fenton KA. Strategies for improving sexual health in ethnic minorities. Curr Opin Infect Dis 2001; 14: 63-69.

4 The One-Stop Shop Evaluation Team. Evaluation of One-Stop Shop Models of Sexual Health Service Provision. London, UK: Department of Health, 2008.

5 McKenzieKJ, Crowcroft NS. Race, ethnicity, culture and science. BMJ 1994; 309: 286-287.

6 McKenzie KJ, Crowcroft NS. Describing race, ethnicity, and culture in medical research. BMJ 1996; 312: 1054.

7 Senior P, Bhopal RS. Ethnicity as a variable in epidemiological research. BMJ 1994; 309: 327-329.

8 Office for National Statistics (ONS). Social Focus in Brief: Ethnicity 2002. London, UK: ONS, 2002.

9 Rankin J, Bhopal R. Current census categories are not good match for identity. BMJ 1999; 318: 1696.

10 Bhattacharyya G, Ison L, Blair M. Minority Ethnic Attainment and Participation in Education and Training: The Evidence. A report for the Department for Education and Skills (DFES). Report Topic Paper RTP01-03. London, UK: DFES, 2003

11 Health Survey for England. The Health of Minority Ethnic Groups. London, UK: Department of Health, 1999.

12 Testa A, Coleman L. Sexual Health Knowledge, Attitudes and Behaviours Among Black and Minority Ethnic Youth in London. A Summary of Findings. London, UK: Trust for the Study of Adolescence and Naz Project London, 2006.

13 Sinha S, Curtis K, Jayakody A, Viner R, Roberts $H$, and the Research with East London Adolescents Community Health Survey (RELACHS). Starting Sex in East London: Protective and Risk Factors for Starting to have Sex Amongst Black and Minority Ethnicity Young People in East London. Paper 1 prepared for the Teenage Pregnancy Unit, 2005.

14 French RS, Joyce L, Fenton K, et al. Exploring the Attitudes and Behaviours of Bangladeshi, Indian and Jamaican Young People in Relation to Reproductive and Sexual Health. Final report to the Teenage Pregnancy Unit, 2005.

15 Griffiths C, French RS, Kanwal-Patel H, Rait G. "Always between two cultures" - young British Bangladeshis and their mothers' views on sex and relationships: implications for developing sexual health education. Cult Health Sex 2008; (in press).

16 Beck A, Majumdar A, Estcourt C, Petrak J. "We don't really have cause to discuss these things, they don't affect us": a collaborative model for developing culturally appropriate sexual health services with the Bangladeshi community of Tower Hamlets. Sex Transm Infect 2005; 81: 158-162.

17 Bradby H, Williams R. Behaviours and expectations in relation to sexual intercourse among 18-20 year old Asians and nonAsians. Sex Transm Infect 1999; 75: 162-167.

18 Wellings K, Nanchahal K, Macdowall W, McManus S, Erens B, Mercer C, et al. Sexual behaviour in Britain: early heterosexual experience. Lancet 2001; 358: 1843-1850.

19 Hennink M, Diamond I, Cooper P. Young Asian women and relationships: traditional or transitional? Ethn Racial Stud 1999; 22: 867-891.

20 Jayakody A, Sinha S, Curtis K, Roberts H, Viner R, and the Research with East London Adolescents Community Health Survey (RELACHS). Culture, Identity, Religion and Sexual Behaviour Among Black and Minority Ethnic Teenagers in East London. Paper 4 prepared for the Teenage Pregnancy Unit, 2005.

21 Saxena S, Oakeshott P, Hilton S. Contraceptive use among South Asian women attending general practices in southwest London. Br J Gen Pract 2002; 52: 392-394.

22 Elam G, Fenton K, Johnson A, Nazroo J, Ritchie J. Exploring Ethnicity and Sexual Health. London, UK: SCPR, 1999.

23 Research with East London Adolescents Community Health Survey (RELACHS). Methods paper prepared for the Teenage Pregnancy Unit, 2005.

24 Elam G, Fenton KA. Researching sensitive issues and ethnicity: lessons from sexual health. Ethn Health 2003; 8: 15-27.

25 Cliffe S, Mortimer J, McGarrigle C, Boisson E, Parry JV, Turner $A$, et al. Surveillance for the impact in the UK of HIV epidemics in South Asia. Ethn Health 1999; 4(1/2): 5-18.

26 Fenton KA, Mercer $\mathrm{CH}$, McManus S, Erens B, Wellings $\mathrm{K}$, Macdowall W, et al. Ethnic variations in sexual behaviour in Great Britain and risk of sexually transmitted infections: a probability survey. Lancet 2005; 365: 1246-1255.

27 Skinner CJ, Saulsbury NKG, Goh BT. Sexually transmitted infections in Bangladeshis resident in the UK: a case-control study. Sex Transm Infect 2002; 78: 120-122.

28 Richardson D, Wood K, Goldmeier D. A qualitative pilot study of Islamic men with lifelong premature (rapid) ejaculation. J Sex Med 2006; 3: 337-343.

29 Richardson D, Goldmeier D. Premature ejaculation - does country of origin tell us anything about etiology? J Sex Med 2005; 2: 508-512.

30 Steggall MJ, Pryce A, Fowler CG. Is ethnicity and religion an aetiological factor in men with rapid ejaculation? Sexual and Relationships Therapy 2006; 21: 429-437.

31 Sumathipala A, Siribaddana SH, Bhugra D. Culture bound syndromes: the story of dhat syndrome. Br J Psychiatry 2004; 184: 200-209.

32 Weston $\mathrm{HJ}$. Public honour, private shame and HIV: issues affecting sexual health service delivery in London's South Asian communities. Health Place 2003; 9: 109-117.

33 Sethi G, Lacey CJ, Fenton KA, Williams IG, Fox E, Sabin CA et al. South Asians with HIV in London: is it time to rethink sexual health service delivery to meet the needs of heterosexual ethnic minorities? Sex Transm Infect 2004; 80: 74-75. 
34 Dougan S, Elford J, Rice B, Brown AE, Sinka K, Evans BG, et al. Epidemiology of HIV among black and minority ethnic men who have sex with men in England and Wales. Sex Transm Infect 2005; 81: 345-350.

35 Teenage Pregnancy Unit, Department of Health. Guidance for Developing Contraception and Sexual Health Advice Services to Reach Black and Minority Ethnic (BME) Young People. London, UK: Department of Health, 2002.

36 Medical Foundation for AIDS \& Sexual Health (MedFASH). National Recommended Standards for Sexual Health Services. London, UK: MedFASH, 2005.

37 Mcavoy BR. Contraceptive services for Asian women in the UK - a review. Fam Pract 1990; 7: 60-64.

38 Raleigh VS, Almond C, Kiri V. Fertility and contraception among ethnic minority women in Great Britain. Health Trends 1997; 29: 109-113.

39 Saxena S, Copas AJ, Mercer C, Johnson AM, Fenton K, Eren $\mathrm{B}$, et al. Ethnic variations in sexual activity and contraceptive use: national cross-sectional survey. Contraception 2006; 74 224-233.

40 Office for National Statistics. Census 2001. London, UK: HMSO, 2001.

41 Rashid J. Contraceptive use among Asian women. Br J Fam Plann 1983; 8: 132-135.

42 Zaklama MS. The Asian community in Leicester and the family planning services. Biol Soc 1984; 1: 63-69.

43 McAvoy BR, Raza R. Asian women: (i) Contraceptive knowledge, attitudes and usage. (ii) Contraceptive services and cervical cytology. Health Trends 1988; 20: 11-17.

44 Garg M. Uptake of family planning services among an ethnically mixed population in a general practice setting. $\mathrm{Br} J$ Fam Plann 1998; 24: 82-83.

45 Hennink M, Diamond I, Cooper P. Contraceptive use dynamics of Asian women in Britain. J Biosoc Sci 1999; 31: 537-554.

46 Hennink M, Cooper P, Diamond I. Asian women's use of family planning services. Br J Fam Plann 1998; 24: 43-52.

47 Hewison J, Green JM, Ahmed S, Cuckle HS, Hirst J, Hucknall $\mathrm{C}$, et al. Attitudes to prenatal testing and termination of pregnancy for fetal abnormality: a comparison of white and Pakistani women in the UK. Prenat Diagn 2007; 27: 419-430.

48 Eskild A, Nesheim BI, Busund B, Vatten L, Vangen S. Childbearing or induced abortion: the impact of education and ethnic background. Population study of Norwegian and Pakistani women in Oslo, Norway. Acta Obstet Gynecol Scand 2007; 86: 298-303.

49 Silverman JG, Gupta J, Decker MR, Kapur N, Raj A. Intimate partner violence and unwanted pregnancy, miscarriage, induced abortion, and stillbirth among a national sample of Bangladeshi women. Br J Obstet Gynaecol 2007; 114: 1246-1252.

50 Tariq S, Edwards SG, Nalabanda A, Ward H, Allen E, Fenton K, et al. Sexual health services for South Asians in London: a case-control study. Int J STD AIDS 2007; 18: 563-564.

51 Ross JDC, Copas A, Stephenson J, Fellows L, Gilleran G. Public involvement in modernising genitourinary medicine clinics: using general public and patient opinion to influence models of service delivery. Sex Transm Infect 2006; 82: 484-488.

52 Baraitser P. Family planning and sexual health: understanding the needs of South Asian women in Glasgow. J Ethn Migr Stud 1999; 25: 133-149.

53 fpa (Family Planning Association). www.fpa.org.uk

54 Naz Project London. www.naz.org.uk.

55 Teenage Pregnancy Unit, Department of Health. Diverse Communities: Identity and Teenage Pregnancy, A Resource for Practitioners. London, UK: Department of Health, 2002.

\section{NEWS ROUNDUP}

\section{Lessons in life}

The emphasis on personal health and sexual education in schools is directed towards avoiding teenage pregnancy. However few young people realise the reasons for infertility in later life. Lisa Jardine, the new head of the Human Fertilisation and Embryology Authority (HFEA), feels this is something young women should be warned about. She wants children to be taught about infertility at school as part of their sex education. "If one in seven of us in the modern world is going to have problems with infertility", she says, "then instead of all the teaching at school being about how to stop getting pregnant, someone had better start teaching about how you do get pregnant, because there are going to be a lot of extremely disappointed people out there",

This is something that Clare Brown, Chief Executive of Infertility Network UK and More To Life, has long advocated: "There is a perception that IVF treatment will fix any fertility problems but it doesn't work for everyone. We need to start telling children at an early age that their fertility is precious and that they need to be aware of the possibility that when they are ready to try for a family it may not be that easy".

National Infertility Day (on 19 July 2008 this year) is a unique event aimed at raising awareness of infertility and all the surrounding issues and providing support and information to patients, their friends and families. It provides delegates with an opportunity not only to gather information but also to discuss infertility issues in general.

For more information on National Infertility Day 2010 and to book online visit www.nationalinfertilityday.com or visit the national charity's website at www.infertility networkuk.com. For support on involuntary childlessness visit www.moretolife.co.uk. Alternatively call the new freephone telephone number 08000087464 .

\section{Vote USA 2008 issues: abortion}

Abortion could be one of the key deciders in the forthcoming American presidential election. Roe v Wade, the 1973 Supreme Court ruling that established a constitutional right to abortion, continues to fuel controversy. A recent poll has shown that $51 \%$ of Americans believe abortion should be available in most cases and $46 \%$ who say it should be illegal. The Democrat presidential candidate, Senator Barack Obama is committed to appointing judges who would not overturn Roe v Wade. The Republican nominee, Senator John McCain, has switched from supporting Roe $\mathrm{v}$ Wade to now saying it should be overturned. Despite having anti-abortion lobbyist, Sarah Palin, as his running mate McCain is likely to focus on the economy, health care and Iraq.

Source: http://news.bbc.co.uk/1/hi/world/americas/7150660.stm

\section{ED due to work-related stress}

Work-related stress may cause erectile dysfunction (ED) in up to 1 in 10 men. Of the $54 \%$ of these men who would consult their doctor about this, the average delay is 17 months from the onset of symptoms. Dr David Edwards, an Oxfordshire GP, comments: "Workplace stress clearly has a strong impact on the incidence of ED in men. Men should think about the underlying cause of their ED, including how to combat their stress levels; not staying too late at work, a balanced and healthy diet, and regular exercise". The SortED in 10 Campaign sponsored by Bayer Schering Pharma, supported the research and highlighted that erection problems are very common, affecting half of all men aged between 40 and 70 years. Men should ask their GP or nurse about what help is available. Oral treatment for ED is only available on the NHS for certain medical conditions.

\section{Source: www.sortEDin10.co.uk}

\section{Books on prescription}

Medication has an important role in helping people with emotional problems, and is often very effective. However, as recommended by the National Institute for Health and Clinical Excellence (NICE), people can also be helped by reading appropriate 'self-help' material. Many self-help books have been shown to be very useful in helping people to overcome a range of psychological problems. The use of books as a means of providing psychological therapy is known as 'bibliotherapy'. In 2003, Neil Frude from Cardiff and the Vale Trust compiled a list of books highly recommended by their clinicians and launched the award-winning Cardiff Book Prescription Scheme aimed at helping people with mild to moderate psychological problems. It allows highly recommended self-help books to be prescribed by GPs, Practice Counsellors, Community Mental Health Teams and other health providers. A 'prescription' for the recommended book is issued by a health professional and can be exchanged for a book (taken from a list of 33 books covering 20 of the most common psychological problems - see Booklist) at any local branch library, free of charge. For further information contact your local PCT and/or the following websites:

Booklist for Wales: http://www.nhsdirect.wales. nhs.uk/language/A4 booklist.pdf

Alphabetical list of PCTs: http://www.nhs.uk/ servicedirectories/Pages/PrimaryCareTrustListing. aspx

Source: http://www.nhsdirect.wales.nhs.uk/small/en/home/health information/mentalhealth/bookprescriptionwales

\section{Spermcatcher}

Play "Spermcatcher", part of the new Brook condom campaign, at www.spermcatcher.net. A3 posters and four different coloured postcards are also available, and can be ordered by calling 0870 7503082 .

\section{Contraception for elephants}

Conservation programmes for elephants in Africa have been so successful that numbers need to be restricted. A programme of long-acting reversible contraception has been developed. This video is fascinating, but the size of the transvaginal probe brought tears to my eyes

Source: http://news.nationalgeographic.com/news/2008/06/ 080605-contraception-video-wc.htm

Reviewed by Henrietta Hughes, MRCGP, DFSRH General Practitioner, London, UK 